\title{
STUDY OF LEVER-ARM EFFECT USING EMBEDDED PHOTOGRAMMETRY AND ON-BOARD GPS RECEIVER ON UAV FOR METROLOGICAL MAPPING PURPOSE AND PROPOSAL OF A FREE GROUND MEASUREMENTS CALIBRATION PROCEDURE
}

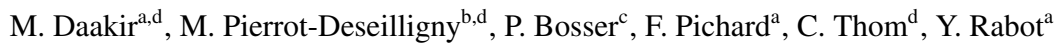 \\ ${ }^{a}$ Vinci-Construction-Terrassement, 1, Rue du docteur Charcot, 91421 Morangis, France - \\ (mehdi.daakir, francis.pichard, yohann.rabot)@ vinci-construction.com \\ buniversité Paris-Est, IGN, ENSG, LOEMI , 6-8 Avenue Blaise Pascal, 77455 Champs-sur-Marne, France - \\ marc.pierrot-deseilligny@ensg.eu \\ ${ }^{c}$ ENSTA Bretagne-OSM Team, 2 rue Francois Verny, 29806 Brest, France - \\ pierre.bosser@ensta-bretagne.fr \\ ${ }^{\mathrm{d} U n i v e r s i t e ́ ~ P a r i s-E s t, ~ I G N, ~ S R I G, ~ L O E M I, ~} 73$ avenue de Paris, 94160 Saint-Mande, France - \\ christian.thom@ign.fr
}

KEY WORDS: UAV, photogrammetry, GPS, lever-arm, calibration, direct-georeferencing, synchronisation, MicMac

\begin{abstract}
:
Nowadays, Unmanned Aerial Vehicle (UAV) on-board photogrammetry knows a significant growth due to the democratization of using drones in the civilian sector. Also, due to changes in regulations laws governing the rules of inclusion of a UAV in the airspace which become suitable for the development of professional activities. Fields of application of photogrammetry are diverse, for instance: architecture, geology, archaeology, mapping, industrial metrology, etc. Our research concerns the latter area. Vinci-ConstructionTerrassement is a private company specialized in public earthworks that uses UAVs for metrology applications. This article deals with maximum accuracy one can achieve with a coupled camera and GPS receiver system for direct-georeferencing of Digital Surface Models (DSMs) without relying on Ground Control Points (GCPs) measurements. This article focuses specially on the lever-arm calibration part. This proposed calibration method is based on two steps: a first step involves the proper calibration for each sensor, i.e. to determine the position of the optical center of the camera and the GPS antenna phase center in a local coordinate system relative to the sensor. A second step concerns a $3 d$ modeling of the UAV with embedded sensors through a photogrammetric acquisition. Processing this acquisition allows to determine the value of the lever-arm offset without using GCPs.
\end{abstract}

\section{INTRODUCTION}

In the context of direct-georeferencing of UAV aerial images using an on-board GPS receiver, lever-arm calibration is an important step in the data processing pipeline to ensure maximum accuracy when generating a DSM. The lever-arm vector is the offset between the GPS receiver antenna phase center and the optical center of the camera. In our configuration, we rely on processing carrier-phase raw embedded GPS data to get the camera position while the photogrammetric image processing based on tie points extraction (Lowe, 2004) provides the camera orientation. The conventional method to calibrate this vector is generally based on external measurements as well as the mechanical stability of the mounting of the sensors on the UAV is required. Indeed, if one of the sensors, the camera or the GPS receiver, exchange position/orientation, calibration results are obviously no longer valid. We propose in the following a study of the impact of leverarm determination on direct-georeferencing accuracy and a different calibration method requiring only photogrammetric measurements without any special mechanical sensors mounting configuration constraint.

\section{HARDWARE}

As part of our research we developed a UAV prototype which can perform, by embedding a camera and a GPS receiver, directgeoreferencing of camera centers in an absolute coordinate sys- tem. This UAV uses home made IGN ${ }^{1}$ LOEM $\left.\right|^{2}$ developed sensors. The choice of the UAV model is intended to be low-cost as for the embedded sensors. The total mass should not exceed $2 \mathrm{Kg}$. Figure 1 shows the UAV used for experiments as well as on-board sensors:
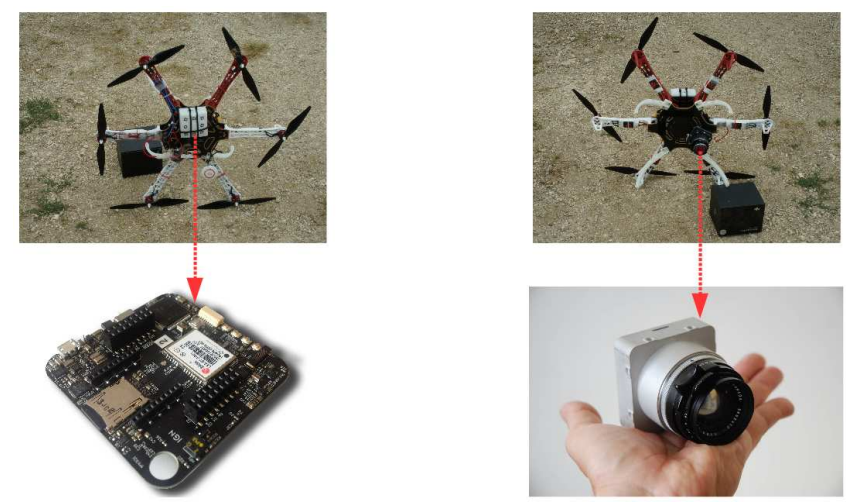

Figure 1: DJI-F550 UAV with IGN GeoCube (left) and on-board light camera (right)

The UAV is the F-550 hexacopter model developed by DJ ${ }^{3}$ It allows to take-off within $0.8 \mathrm{Kg}$ payload but its autonomy still relatively low, about $10 \mathrm{~min}$. The GPS receiver is the GeoCube devel-

\footnotetext{
${ }^{1}$ French Mapping Agency

${ }^{2}$ Opto-Electronics, Instrumentation and Metrology Laboratory

${ }^{3}$ www.dji.com/product/flame-wheel-arf/
} 
oped at IGN. The GeoCube (Benoit et al., 2015) is a multi-sensor instrument that embeds a LEA-6T-001 u-blox (u-blox, 2014) GPS module chip. It records carrier-phase raw measurements on $\mathrm{L} 11^{4}$ band of GPS constellation. The light camera used (Martin et al., 2014) is also developed at $I G N$ and is specially designed for aerial photogrammetry applications using UAVs. This camera has a full frame sensor with a fixed focal length. Its mass does not exceed $300 \mathrm{~g}$.

\section{LEVER-ARM CALIBRATION}

\subsection{Auto-calibration method}

A conventional method for calibrating the lever-arm offset is to acquire GPS data coupled with images over a scene containing GCPs. These GCPs are used to estimate the unknown parameters of the $3 d$ similarity to convert camera poses from bundle block adjustment relative frame to an absolute one. Figure 2 shows an image acquired by a UAV of a scene provided with GCPs which are used for in-flight classical lever-arm calibration.

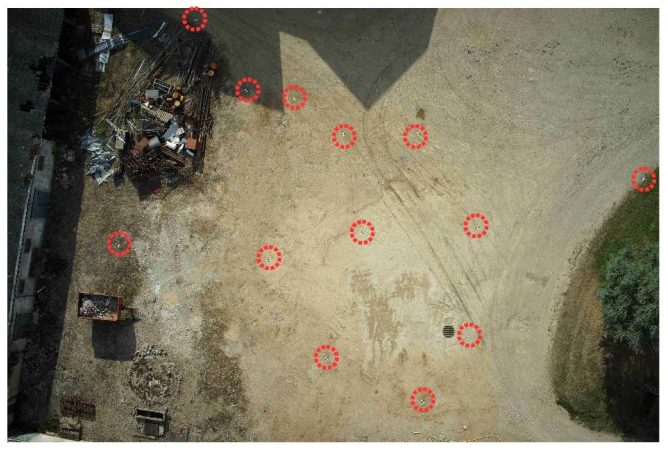

Figure 2: Scene containing GCPs used for lever-arm calibration

These GCPs allow simultaneously to constrain the estimation of the lever-arm offset. In theory one image is needed to estimate the value of this vector. However, in order to qualify the accuracy of this estimation, and supposing that time synchronisation is perfect between both sensors, a least squares adjustment is performed with several dozen images by minimizing the following system:

$$
\left\{\begin{array}{c}
\mathcal{S}\left(\left.\overrightarrow{\mathcal{C}_{k}}\right|_{r}\right)=\left.\overrightarrow{\mathcal{G}_{k}}\right|_{t}+\mathcal{R}_{k} \cdot \overrightarrow{\mathcal{O}} \\
\ldots \cdots
\end{array}\right.
$$

where: $\quad \mathcal{S}=7$ parameters $3 d$ similarity $(\overrightarrow{\mathcal{T}}, \mu, \mathcal{R})$

$\mathcal{R}_{k}=$ orientation of the image $k$

$\overrightarrow{\mathcal{O}}=$ lever-arm vector

$\left.\overrightarrow{\mathcal{G}_{k}}\right|_{t}=$ GPS position of the image $k$

For aerial acquisitions using UAVs, vertical component of global translation $\left.\vec{T}\right|_{z}$ and vertical component of lever-arm vector $\left.\vec{O}\right|_{z}$ are correlated. This correlation is introduced due to the fact that the camera maintains the same orientation during a UAV flight.
We need at least one GCP measurement in order to apply a constraint during the adjustment. This constraint is applied to the estimation of $3 d$ similarity parameters as following:

$$
\left\{\begin{array}{c}
\mathcal{S}\left(\left.\overrightarrow{\mathcal{C}_{k}}\right|_{r}\right)=\left.\overrightarrow{\mathcal{G}_{k}}\right|_{t}+\mathcal{R}_{k} \cdot \overrightarrow{\mathcal{O}} \\
\ldots \cdots \\
\mathcal{S}\left(\left.\overrightarrow{\mathcal{P}_{i}}\right|_{r}\right)=\left.\overrightarrow{\mathcal{P}_{i}}\right|_{t} \\
\cdots \cdots
\end{array}\right.
$$

\subsection{Pseudo-materialization method}

Phase and optical centers can not be directly measured. This calibration method is to make a pseudo-materialization of these. The idea is to determine their relative position with respect to a network of known points on the GPS receiver and the camera in a local frame. Figure 3 shows the targets on sensors.
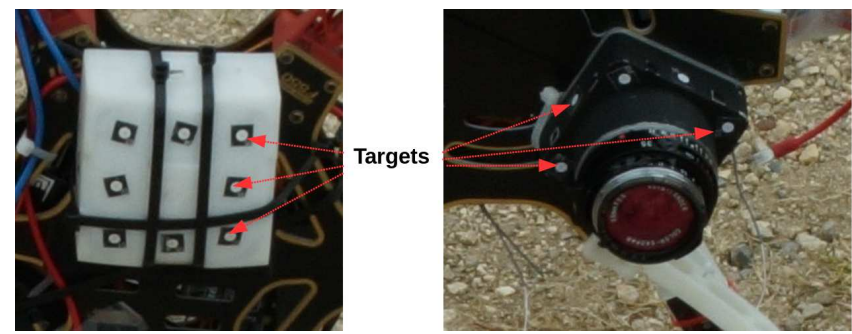

Figure 3: Targets fixed on the sensors

The acquisition of the calibration data protocol, which is carried out only once, is based on the following photogrammetric measurements:

\section{Camera optical center calibration:}

- a photogrammetric acquisition is performed using the camera to calibrate

- the camera to calibrate is positioned on a stable surface

- the camera to calibrate takes a single image when positioned on the stable surface

- a second camera performs a photogrammetric acquisition around the first one

Figure 4 shows a photogrammetric acquisition around the camera to be calibrated while positioned on a stable surface.

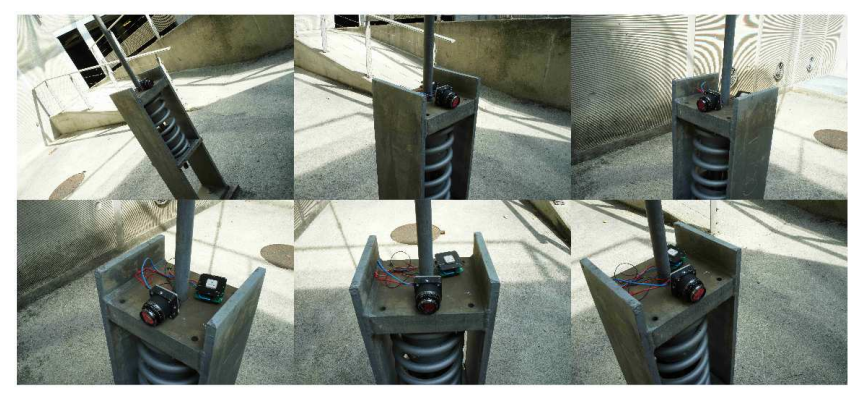

Figure 4: Photogrammetric acquisition around the UAV on-board camera

2. GPS antenna phase center calibration: 
- the GPS receiver is positioned on a scene that contains already known targets in an absolute reference system

- for each GPS observations session, a photogrammetric acquisition is performed

- the GPS receiver performs $\mathrm{a} \sim 90^{\circ}$ rotation and a new session is started coupled to a new photogrammetric acquisition

Figure 5 shows a photogrammetric acquisition of the GPS receiver for a given position.

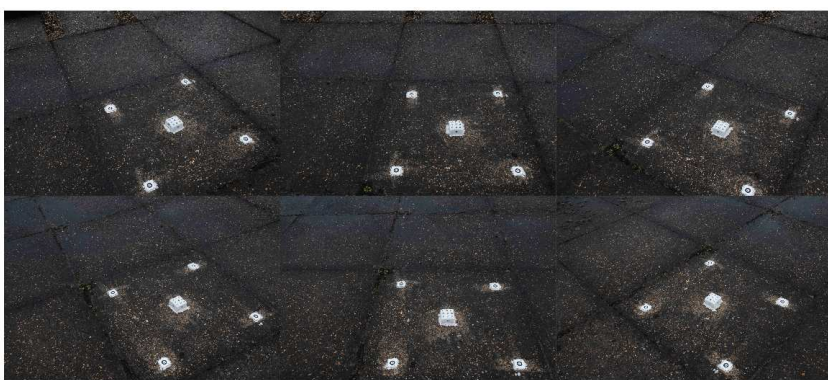

Figure 5: Photogrammetric acquisition of the UAV on-board GPS receiver

3.2.1 Camera optical center calibration As a result of data acquisition step, we have taken two converging photogrammetric acquisitions of the same scene. The first performed with the camera to be calibrated and the second one performed with another camera around the first. A ruler is disposed on the scene because the results of cameras poses estimation must be expressed in a scaled frame. Bundle block adjustment of all acquired images is achieved using Apero (Pierrot-Deseilligny and Clery, 2011) module of the IGN MicMac photogrammetric suite. A convergent acquisition, in the form of a circle, is the most suitable to ensure high overlap rates. Figure 6 shows images acquisition geometry.

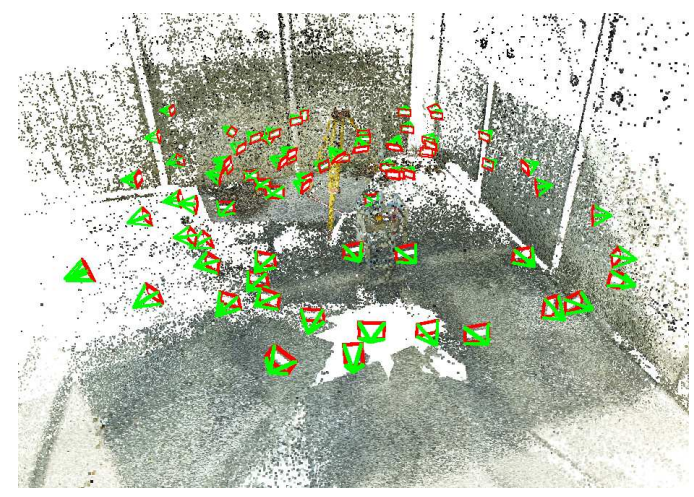

Figure 6: The circular geometry of the acquisition

After performing the bundle block adjustment, the targets $\left.\vec{p}_{i}\right|_{k}$ located on the camera 3 to be calibrated are measured in the images $k$ and their $3 d$ coordinates $\vec{P}_{i}$ estimated by pseudo-intersections. This estimate is carried out by a least squares adjustment of the following system of equations:

$$
\left\{\begin{array}{c}
\cdots \cdots \\
\left.\vec{p}_{i}\right|_{k}-\zeta\left(\pi\left(\mathcal{R}_{k}\left(\vec{P}_{i}-\overrightarrow{\mathcal{C}}_{k}\right)\right)\right)=\overrightarrow{0} \\
\cdots \cdots
\end{array}\right.
$$

where:
- $\left.\vec{p}_{i}\right|_{k}$ vector of $2 d$ image coordinates of target $i$ on image $k$

- $\zeta$ is a $\mathbb{R}^{2} \rightarrow \mathbb{R}^{2}$ application describing internal parameters of the camera

- $\pi$ is a $\mathbb{R}^{3} \rightarrow \mathbb{R}^{2}$ projective application

- $\left(\overrightarrow{\mathcal{C}_{k}}, \mathcal{R}_{k}\right)$ position and orientation of image $k$

- $\vec{P}_{i}$ target $3 d$ position parameter to be estimated

Using the external parameters of the single image $s$ acquired by the camera to be calibrated while placed on the stable surface, the $3 d$ targets positions $\left.\vec{P}_{i}\right|_{r}$ are expressed in the camera frame $\left.\vec{P}_{i}\right|_{\text {cam }}$ using:

$$
\left.\vec{P}_{i}\right|_{\text {cam }}=\mathcal{R}_{s}^{T} \cdot\left(\left.\vec{P}_{i}\right|_{r}-\left.\overrightarrow{\mathcal{C}_{s}}\right|_{r}\right)
$$

where:

- $\left.\vec{P}_{i}\right|_{\text {cam }}$ position of target $i$ expressed in the camera frame

- $\left.\overrightarrow{\mathcal{C}_{s}}\right|_{r}, \mathcal{R}_{s}$ position and orientation of image $s$ expressed in the relative frame

- $\left.\vec{P}_{i}\right|_{r}$ previous estimated $3 d$ position of target $i$ expressed in the relative frame

3.2.2 GPS antenna phase center calibration The calibration of the GPS antenna phase center is based on the same principle. Here, we need to express the targets in an absolute reference system ${ }^{5}$. Photogrammetric acquisitions are performed on a scene where are installed known targets in the same system determined with topometric measurements.

We perform 4 rotations of $\sim 90^{\circ}$ to highlight the relative displacement of the antenna phase center position expressed in the local frame of the receiver. For each rotation $3 h$ GPS raw observations sampled at $1 \mathrm{~Hz}$ are recorded. RTKlib (Takasu, 2011) open-source software is used to perform differential static postprocessing of L1 carrier-phase measurements. Each session gives an estimate of the GPS antenna phase center position. For each rotation, a photogrammetric acquisition is performed. Figure 7 shows images acquisition geometry.

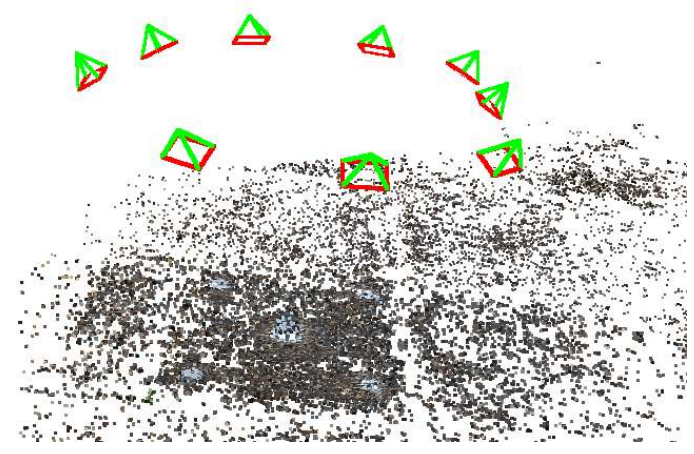

Figure 7: Images acquired around the GPS receiver

The estimated camera poses from bundle block adjustment are expressed in the GPS absolute reference system using 4 GCPs. The targets $\left.\vec{g}_{i}\right|_{k}$ located on the GPS receiver 3 are measured in

${ }^{5}$ GPS World Geodetic System 1984 
the images $k$ and their $3 d$ coordinates $\vec{G}_{i}$ estimated using system of equations 3 . Targets positions and the position of the antenna phase center after the first session of measurements are arbitrarily considered as a reference state. Successive positions are expressed in the frame of the first session by estimating the 6 parameters $\left(R_{i}, \vec{T}_{i}\right)$ of the rigid transformation $\mathcal{D}_{i}$ such that:

$$
\mathcal{D}_{i}\left(\vec{P}_{i}\right)=\vec{P}_{0}
$$

where:

- $\vec{P}_{i}$ position of targets and antenna phase center after session of observations $i$

- $\vec{P}_{0}$ position of targets and antenna phase center after the first session of observations

Figure 8 shows the planimetric displacement of the antenna phase center expressed in the frame of the first session of observations.

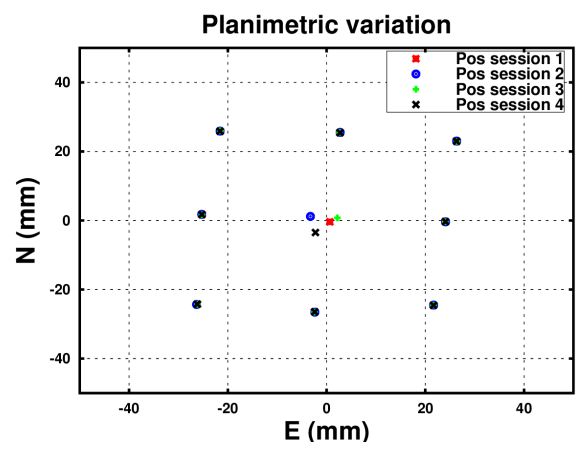

Figure 8: Planimetric variation of the antenna phase center

Symbols at the center of Figure 8 represent the predictions of the antenna phase center for each session. The mean deviation between all sessions is $0.7 \mathrm{~cm}$ and the maximum deviation is 1.1 $\mathrm{cm}$. Final values for the calibration of targets and antenna phase center positions are the average of estimated positions including all observations sessions.

3.2.3 Computing lever-arm offset value To get the value of the lever-arm vector after the calibration of optical and phase centers performed, it is necessary to conduct a photogrammetric acquisition of the UAV, with the sensors mounted, before take-off. Figure 9 shows images and geometry acquisition of the UAV on the ground.

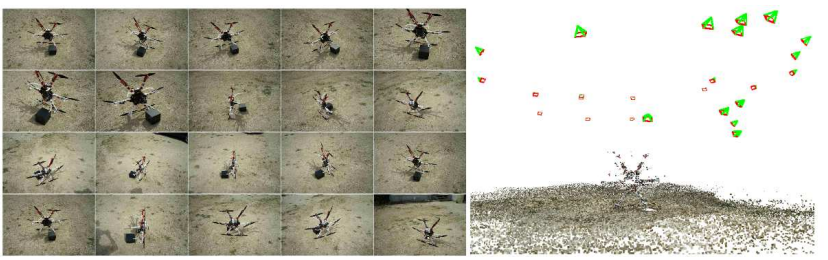

Figure 9: Photogrammetric acquisition around UAV before takeoff (left) and images geometry acquisition (right)

To calculate the lever-arm offset value only image measurements are needed. Let $N$ be the number of targets measured on the camera and $N^{\prime}$ the number of targets measured on the GPS receiver. Let:

- $\left.\vec{v}\right|_{R_{r}}=\left[\left.\vec{P}_{1}\right|_{r}, \ldots,\left.\vec{P}_{N}\right|_{r}\right]$ camera targets estimated positions in the bundle block adjustment relative frame
- $\left.\vec{v}\right|_{R_{\text {cam }}}=\left[\left.\vec{P}_{1}\right|_{c a m}, \ldots,\left.\vec{P}_{N}\right|_{c a m}\right]$ camera targets estimated positions from calibration expressed in the camera frame

- $\left.\overrightarrow{v^{\prime}}\right|_{R_{r}}=\left[\left.\vec{G}_{1}\right|_{r}, \ldots,\left.\vec{G}_{N^{\prime}}\right|_{r}\right]$ GPS targets estimated positions in the bundle block adjustment relative frame

- $\left.\overrightarrow{v^{\prime}}\right|_{R_{l}}=\left[\left.\vec{G}_{1}\right|_{l}, \ldots,\left.\vec{G}_{N^{\prime}}\right|_{l}\right]$ GPS targets estimated positions from calibration expressed in the GPS receiver local frame

- $\left.\overrightarrow{v^{\prime}}\right|_{R_{c}}=\left[\left.\vec{G}_{1}\right|_{c}, \ldots,\left.\vec{G}_{N^{\prime}}\right|_{c}\right]$ GPS targets estimated positions expressed in the camera frame

- $\left.\vec{C}_{g p s}\right|_{R_{l}}$ antenna phase center from calibration expressed in the GPS receiver local frame

- $\vec{O}$ lever-arm vector by definition expressed in the camera frame

To estimate the value of the lever-arm offset $\vec{O}$, one must realize:

1. estimate the 7 parameters $\left(\lambda, R_{1}, \vec{T}_{1}\right)$ of the $3 d$ similarity $\mathcal{S}$ such that camera poses are expressed in the calibrated camera frame:

$$
\mathcal{S}\left(\left.\vec{v}\right|_{R_{r}}\right)=\left.\lambda \cdot R_{1} \cdot \vec{v}\right|_{R_{c a m}}+\vec{T}_{1}
$$

2. express GPS targets in the camera frame:

$$
\left.\overrightarrow{v^{\prime}}\right|_{R_{c}}=\mathcal{S}\left(\left.\overrightarrow{v^{\prime}}\right|_{R_{r}}\right)
$$

3. estimate the 6 parameters $\left(R_{2}, \vec{T}_{2}\right)$ of the rigid transformation $\mathcal{D}_{i}$ such that:

$$
\mathcal{D}\left(\left.\overrightarrow{v^{\prime}}\right|_{R_{l}}\right)=\left.R_{2} \cdot \overrightarrow{v^{\prime}}\right|_{R_{c}}+\vec{T}_{2}
$$

4. express the lever-arm offset value as:

$$
\vec{O}=\left.R_{2} \cdot \vec{C}_{g p s}\right|_{R_{l}}+\vec{T}_{2}
$$

\section{RESULTS}

A data acquisition with the system as presented in 1 has been made. Two photogrammetric acquisitions are performed as shown in Figure 9. The first one before UAV take-off and the second one after landing. Each acquisition provides a lever-arm value with respect to the second calibration methodology 3.2 and allows us at least to control the mechanical stability between the start of the acquisition and its end.

Before comparing the values of the lever-arm vector provided by this method and the one obtained by classical in-flight selfcalibration method based on GCPs 3.1. we can compare the calculated values before the UAV take-off and after the UAV landing. Table 1 gives lever-arm values for both measurements from two photogrammetric acquisitions of the UAV on the ground and the in-flight estimated value using all available GCPs (17) during this experiment.

\begin{tabular}{|c|c|c|c|}
\hline Method & $X_{c}(\mathrm{~mm})$ & $Y_{c}(\mathrm{~mm})$ & $Z_{c}(\mathrm{~mm})$ \\
\hline Before UAV take-off & 86 & -52 & -82 \\
After UAV landing & 85 & -51 & -81 \\
In-flight calibration & 98 & -70 & -91 \\
\hline
\end{tabular}

Table 1: Different estimates of values of the lever-arm offset

Both photogrammetric acquisitions of the UAV give two leverarm values within $2 \mathrm{~mm}$ difference. It proves firstly that the 
mechanical assembly is stable and the sensors keep a fixed relative positions while the difference between the two calibration techniques is $26 \mathrm{~mm}$ and is more significant.

As stated above, it is possible to estimate the lever-arm offset without adding a constraint equation using one or several GCPs. Figure 10 shows the variation of the lever-arm vector estimation value based on the number of used GCPs during a least squares adjustment for the in-flight self-calibration method.
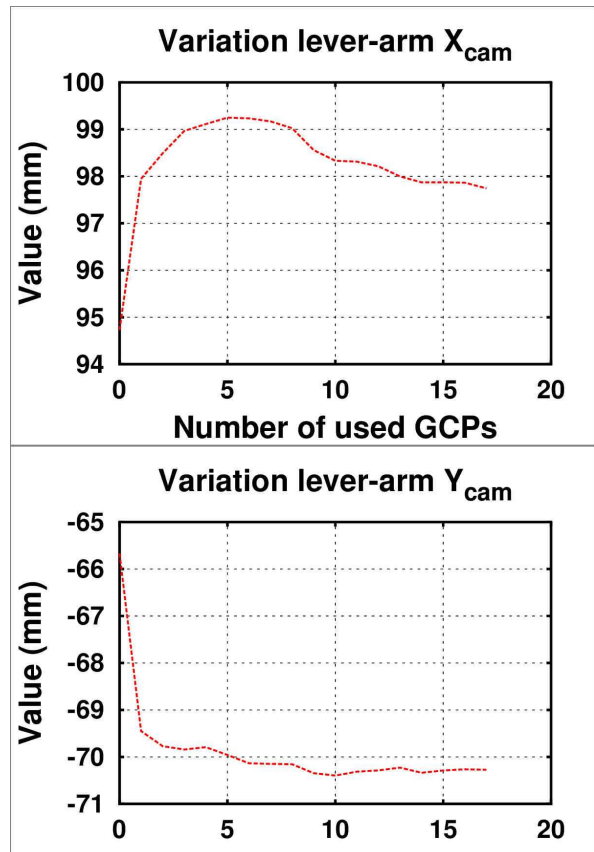

Number of used GCPs

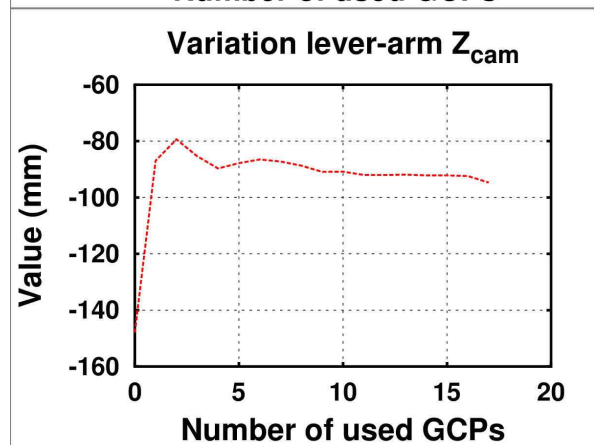

Figure 10: Variation of the lever-arm estimation value using 0 to 17 GCPs

The curves of the axial components show a convergence of the parameter estimation with a stability which increases with the number of GCPs used. However, the difference between the two lever-arm calibration methods seems high $(26 \mathrm{~mm})$.

To study the impact of this difference a second flight is made to have independent data sets. The first estimated lever-arm values given in Table 1 are used to correct GPS post-processed trajectory to perform a direct-georeferencing of camera centers. The residual on check points is calculated using camera poses based on GPS data. Figure 11 gives a comparison on residuals computed on check points for each lever-arm calibration method.

Figure 11 shows that residuals are most important for the calibration method based on a photogrammetric acquisition of the UAV on the ground. Residuals for this method give an accuracy of $2 \mathrm{~cm} \pm 1.4 \mathrm{~cm}$ while the in-flight calibration method gives an

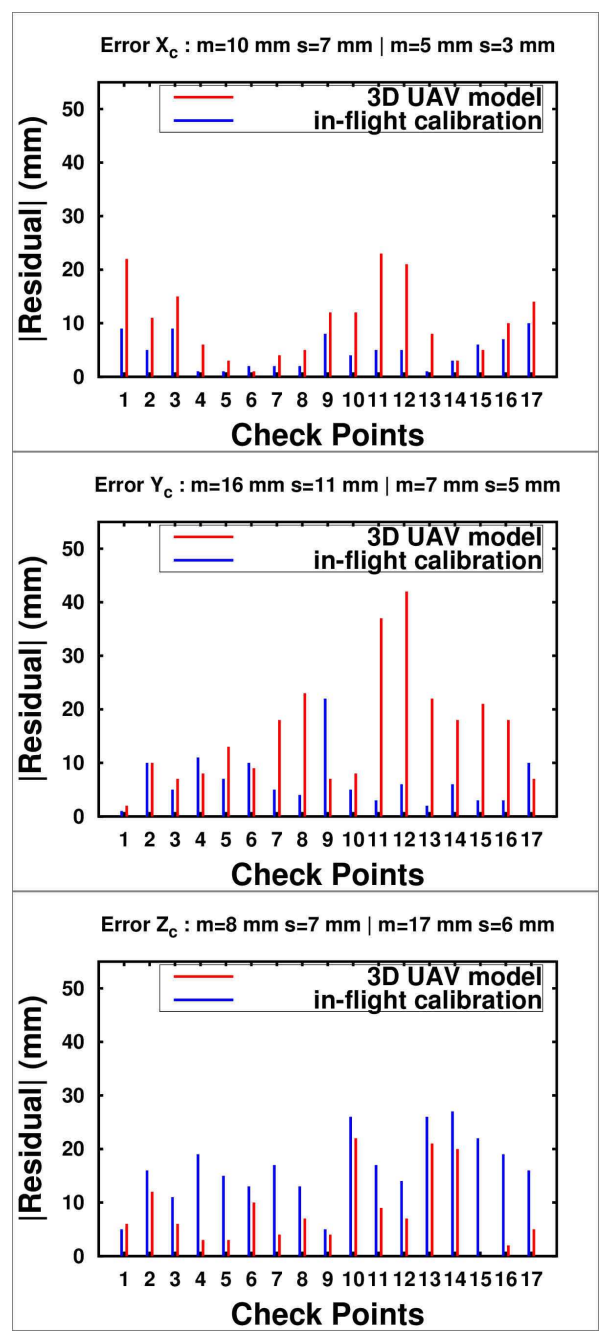

Figure 11: Comparison of absolute residuals using two different lever-arm calibration techniques

accuracy of $1.8 \mathrm{~cm} \pm 0.8 \mathrm{~cm}$. However, the second method requires GCPs and a stable mechanical mounting of sensors on the UAV to be reused while the first one needs only image measurements and requires no special mechanical configuration.

The difference in accuracy between the two methods is due to the fact that, for the photogrammetric acquisition of the UAV method, the calibration of the antenna phase center is not entirely correct. Indeed, this calibration, which requires making static observations and photogrammetric acquisitions of the GPS receiver, is performed on the ground. However, the antenna phase center position is correlated with the antenna environment through the multipath effect. The position of the antenna phase center in the local frame of the GPS receiver finds itself biased because of the multipath effect.

Given the fact that the multipath effect is negligible for an onboard antenna on a UAV during a flight, this bias introduced during phase antenna center calibration can be estimated by computing the difference between the values of the lever-arm, the one estimated in-flight and the one estimating with a photogrammetric acquisition of the UAV on the ground. 


\section{FURTHER WORK}

In order to validate the lever-arm proposed calibration method, it is necessary to conduct a second experiment where two independent datasets will be acquired. The difference here is that the position and the orientation of the camera must be different. The first flight will be used to correct the value of the measured leverarm on the ground from the multipath effect and the second flight will determine whether this correction improves the accuracy on check points in comparison to the results provided above.

\section{CONCLUSIONS}

We propose in this article a study about a method of estimating the lever-arm offset in the context of direct-georeferencing of a close-range photogrammetric acquisition performed with a UAV. The proposed method is flexible because requires no mechanical constraint for the mounting of the sensors on the UAV on each flight. Once the optical and antenna phase centers calibrated, the estimation of the lever-arm value is based solely on photogrammetric measurements across a photogrammetric images acquisition of the UAV on the ground. Also, this method allows to control the stability of the sensors mounting between different flights. The accuracy achieved by this method is lower than the conventional in-flight estimation method. During our experiments we achieved an absolute accuracy of $2 \mathrm{~cm} \pm 1.4 \mathrm{~cm}$ on check points while the classical method achieved an absolute accuracy of 1.8 $\mathrm{cm} \pm 0.8 \mathrm{~cm}$. However, this second method requires having external measurements (GCPs) and that the mechanical configuration remains the same from one flight to another that it may be still valid.

\section{REFERENCES}

Benoit, L., 2014. Positionnement GPS précis et en temps-réel dans le contexte de réseaux de capteurs sans fil type Geocube : application à des objets géophysiques de taille kilométrique. $\mathrm{PhD}$ thesis, École Normale Supérieure.

Benoit, L., Briole, P., Martin, O., Thom, C., Malet, J.-P. and Ulrich, P., 2015. Monitoring landslide displacements with the geocube wireless network of low-cost gps. Engineering Geology 195, pp. 111-121.

Bláha, M., Eisenbeiss, H., Grimm, D. and Limpach, P., 2011. Direct georeferencing of uavs. ISPRS - International Archives of the Photogrammetry, Remote Sensing and Spatial Information Sciences XXXVIII-1/C22, pp. 131-136.

Chiang, K.-W., Tsai, M.-L. and Chu, C.-H., 2012. The development of an uav borne direct georeferenced photogrammetric platform for ground control point free applications. Sensors 12(7), pp. 9161-9180.

Cramer, M., Bovet, S., Gültlinger, M., Honkavaara, E., McGill, A., Rijsdijk, M., Tabor, M. and Tournadre, V., 2013. On the use of rpas in national mapping ; the eurosdr point of view. ISPRS - International Archives of the Photogrammetry, Remote Sensing and Spatial Information Sciences XL-1/W2, pp. 93-99.

Daakir, M., Pierrot-Deseilligny, M., Bosser, P., Pichard, F. and Thom, C., 2015. Uav onboard photogrammetry and gps positionning for earthworks. ISPRS - International Archives of the Photogrammetry, Remote Sensing and Spatial Information Sciences XL-3/W3, pp. 293-298.

DJI, 2015. F550 user manual.

Fraser, C. S., 1997. Digital camera self-calibration. ISPRS Journal of Photogrammetry and Remote sensing 52(4), pp. 149-159.

Fraser, C. S., 2013. Automatic camera calibration in close range photogrammetry. Photogrammetric Engineering \& Remote Sensing 79(4), pp. 381-388.

Gruen, A., 2012. Development and status of image matching in photogrammetry. The Photogrammetric Record 27(137), pp. 36-57.

Klobuchar, J. et al., 1987. Ionospheric time-delay algorithm for singlefrequency gps users. Aerospace and Electronic Systems, IEEE Transactions on (3), pp. 325-331.

Lowe, D. G., 2004. Distinctive image features from scale-invariant keypoints. International journal of computer vision 60(2), pp. 91-110.
Martin, O., Meynard, C., Pierrot-Deseilligny, M., Souchon, J. and Thom, C., 2014. Réalisation d'une caméra photogrammétrique ultralégère et de haute résolution. http://drone.teledetection.fr/.

Pfeifer, N., Glira, P. and Briese, C., 2012. Direct georeferencing with on board navigation components of light weight uav platforms. ISPRS - International Archives of the Photogrammetry, Remote Sensing and Spatial Information Sciences XXXIX-B7, pp. 487-492.

Pierrot-Deseilligny, M., 2015. MicMac, Apero, Pastis and Other Beverages in a Nutshell!

Pierrot-Deseilligny, M. and Clery, I., 2011. Apero, an open source bundle adjusment software for automatic calibration and orientation of set of images. ISPRS - International Archives of the Photogrammetry, Remote Sensing and Spatial Information Sciences XXXVIII-5/W16, pp. 269276.

Pierrot-deseilligny, M. and Paparoditis, N., 2006. A multiresolution and optimization-based image matching approach: An application to surface reconstruction from spot5-hrs stereo imagery. In: In: Proc. of the ISPRS Conference Topographic Mapping From Space (With Special Emphasis on Small Satellites), ISPRS.

Rehak, M., Mabillard, R. and Skaloud, J., 2013. A micro-uav with the capability of direct georeferencing. ISPRS - International Archives of the Photogrammetry, Remote Sensing and Spatial Information Sciences XL-1/W2, pp. 317-323.

Rieke, M., Foerster, T., Geipel, J. and Prinz, T., 2011. High-precision positioning and real-time data processing of uav-systems. International Archives of Photogrammetry, Remote Sensing and Spatial Information Sciences 38, pp. 1-C22.

Saastamoinen, J., 1972. Atmospheric correction for the troposphere and stratosphere in radio ranging satellites. The use of artificial satellites for geodesy pp. 247-251

Stempfhuber, W. and Buchholz, M., 2011. A precise, low-cost rtk gnss system for uav applications. ISPRS - International Archives of the Photogrammetry, Remote Sensing and Spatial Information Sciences XXXVIII-1/C22, pp. 289-293.

Takasu, T., 2011. Rtklib: An open source program package for gnss positioning.

Tournadre, V., Pierrot-Deseilligny, M. and Faure, P. H., 2014. Uav photogrammetry to monitor dykes - calibration and comparison to terrestrial lidar. ISPRS - International Archives of the Photogrammetry, Remote Sensing and Spatial Information Sciences XL-3/W1, pp. 143-148.

Tournadre, V., Pierrot-Deseilligny, M. and Faure, P. H., 2015. Uav linear photogrammetry. ISPRS - International Archives of the Photogrammetry, Remote Sensing and Spatial Information Sciences XL-3/W3, pp. 327333.

Tsai, M., Chiang, K., Huang, Y., Lin, Y., Tsai, J., Lo, C., Lin, Y. and Wu, C., 2010. The development of a direct georeferencing ready uav based photogrammetry platform. In: Proceedings of the 2010 Canadian Geomatics Conference and Symposium of Commission I.

Turner, D., Lucieer, A. and Wallace, L., 2014. Direct georeferencing of ultrahigh-resolution uav imagery. Geoscience and Remote Sensing, IEEE Transactions on 52(5), pp. 2738-2745.

u-blox, 2009. GPS Antennas RF Design Considerations for u-blox GPS Receivers. u-blox AG.

u-blox, 2013. u-blox 6 Receiver Description Including Protocol Specification. u-blox AG.

u-blox, 2014. LEA-6 u-blox 6 GPS Modules Data Sheet Objective Specification. u-blox AG. 